For reprint orders, please contact: reprints@futuremedicine.com

\section{Our panel of experts highlight the most important research articles across the spectrum of topics relevant to the field of lung cancer management}

\section{Lung Cancer Management}

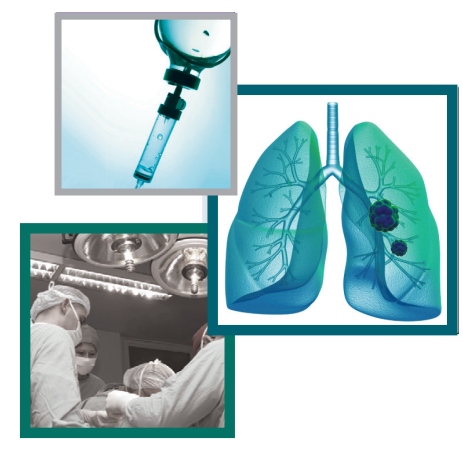

Expert panel: Antonio Rossi, S.G. Moscati Hospital, Avellino, Italy; Minal Kale, Mount Sinai Hospital, New York, NY, USA; Juan Wisnivesky, Mount Sinai Hospital, New York, NY, USA

Langer CJ, Novello S, Park K et al. Randomized, Phase III trial of first-line figitumumab in combination with paclitaxel and carboplatin versus paclitaxel and carboplatin alone in patients with advanced non-smallcell lung cancer. J. Clin. Oncol. 32(19), 2059-2066 (2014).

A multicenter, randomized, Phase III trial compared figitumumab (CP-751,871, at the dose of $20 \mathrm{mg} / \mathrm{kg}$ ), a fully human immunoglobulin G2 monoclonal antibody inhibiting IGF-1R, plus paclitaxel $\left(200 \mathrm{mg} / \mathrm{m}^{2}\right)$ and carboplatin (area under the concentration-time curve, $6 \mathrm{mg} \mathrm{min} / \mathrm{ml}$ ) with chemotherapy alone once every 3 weeks for up to six cycles, as first-line treatment in patients with advanced non-small-cell lung cancer (NSCLC). The primary end point was overall survival (OS). The study was closed early by an independent Data Safety Monitoring Committee because of futility and an increased incidence of serious adverse events (66 and 51\%; $p<0.01$ ) and treatment-related deaths ( 5 vs $1 \%$; $\mathrm{p}<0.01$ ) with figitumumab. A total of 681 patients were randomized and 671 received treatment. Median OS was 8.6 months for figitumumab plus chemotherapy and 9.8 months for chemotherapy alone (hazard ratio [HR]: 1.18, 95\% CI: 0.99-1.40; $\mathrm{p}=0.06$ ); median progression-free survival was 4.7 and 4.6 months, respectively (HR: $1.10 ; \mathrm{p}=0.27$ ); the objective response rates were 33 and $35 \%$, respectively.

Despite interesting results reported by Phase II trials, figitumumab failed to show, in the subsequent Phase III randomized trials, any outcome improvements in the management of advanced NSCLC. Unfortunately, this is a further evidence of the failure of the clinical development of a targeted agent. It is important to have a target for a new biological agent but it is also important to understand whether NSCLC is really addicted to that target in order to use the specific inhibitor. Thus, further clinical developments of this type with figitumumab in advanced NSCLC are not recommended.

- Article written by Antonio Rossi

Kawaguchi T, Ando M, Asami K et al. Randomized Phase III trial of erlotinib versus docetaxel as secondor third-line therapy in patients with advanced non-small-cell lung cancer: Docetaxel and Erlotinib Lung Cancer Trial (DELTA). J. Clin. Oncol. 32, 1902-1908 (2014).

This Phase III trial investigated the efficacy of erlotinib versus docetaxel in patients with advanced non-small-cell lung cancer (NSCLC) previous treated with one or two chemotherapy regimens and not selected for the EGF mutation status. The primary end point was progressionfree survival (PFS) while secondary end points included overall survival (OS), response rate, safety and analyses on $E G F R$ wild-type (WT) tumors. A total of 301 patients were randomly assigned to erlotinib (150 mg daily; $\mathrm{n}=150)$ and docetaxel $\left(60 \mathrm{mg} / \mathrm{m}^{2}\right.$ every 3 weeks; $\left.\mathrm{n}=151\right)$.
Future $\because$
Medicine
part of 
Median PFS was 2.0 months for erlotinib and 3.2 months for docetaxel (hazard ratio [HR]: 1.22; 95\% CI: 0.97-1.55; p = 0.09). Median OS was 14.8 versus 12.2 months (HR: 0.91; 95\% CI: $0.68-1.22 ; \mathrm{p}=0.53$ ), respectively. In a subset analysis of EGFR WT tumors, 109 and 90 patients in the erlotinib and docetaxel groups, respectively, PFS for erlotinib versus docetaxel was 1.3 and 2.9 months (HR: 1.45; 95\% CI: $1.09-1.94 ; \mathrm{p}=0.01)$, and $\mathrm{OS}$ was 9.0 versus 10.1 months (HR: 0.98; 95\% CI: 0.69-1.39; $\mathrm{p}=0.91)$, respectively. The most common adverse event with erlotinib was rash (92.7\%), whereas docetaxel was associated with fatigue $(71.3 \%)$, nausea $(50.0 \%)$ and hematologic toxicities.

This study confirmed that there are no efficacy differences between erlotinib and docetaxel in EGFR-unselected NSCLC pretreated patients. A PFS significant advantage for docetaxel was reported in the subgroup of EGFR WT patients despite no OS difference was showed. However, this is a subset analysis which supports the lower efficacy of erlotinib in this subgroup of patients as reported by prospective randomized trials.

We do not need more trials investigating targeted agents in biological unselected NSCLC patients, the new era of research should be performed with studies focused on NSCLC oncogene-addicted patients to develop the corresponding inhibitors in order to establish a large treatment benefit.

\section{- Article written by Antonio Rossi}

Soares M, Toffart AC, Timsit JF et al.; for
the Lung Cancer in Critical Care (LUCCA)
Study Investigators. Intensive care in
patients with lung cancer: a multinational
study. Ann. Oncol. 25(9), 1829-1835 (2014).

The characteristics and clinical outcomes of lung cancer patients requiring admission to an intensive care unit (ICU) are not well understood. In this multinational, prospective study conducted in 22 ICUs in South America and Europe the characteristics of first-time ICU admissions among 449 lung cancer patients (88\% nonsmall-cell type) were evaluated. Patients were followed until hospital discharge, and hospital survivors were contacted 6 months after ICU admission. The main reasons for ICU admission were postoperative complications (41\%) followed by acute respiratory failure (23\%).
Over half (53\%) of patients required ventilator support on day 1 of admission and the median length of stay was 4 (2-10) days. A minority of patients $(6 \%)$ received emergency anticancer treatments, including chemotherapy and radiation therapy. Among all patients, the in-ICU mortality was $28 \%$, compared with the in-hospital mortality of $39 \%$. The 30 - and 60-day mortality rates were 41 and 55\%, respectively. High-volume centers had lower mortality rates. Poor performance status was an independent determinant of 30-day and 6-month mortality.

This observational study sheds light on the clinical characteristics and outcomes of lung cancer patients requiring ICU admission. The study benefits from the level of details captured about each patient, such as performance status. Additionally, the results suggest that despite a high mortality rate, in addition to patient characteristics, system-level factors such as a patient volume are important.

- Article written by Minal Kale \& Juan Wisnivesky

Kris MG, Johnson BE, Berry LD et al. Using multiplexed assays of oncogenic drivers in lung cancers to select targeted drugs. JAMA 311(19), 1998-2006 (2014).

The presence of EGFR gene mutations, in non-small-cell lung cancer (NSCLC) has been shown to predict clinical response to EGFRtyrosine kinase inhibitors. Although the mutations are only found in a minority of patients, treatment with tyrosine kinase inhibitors have resulted in improved survival. In this multicenter study from 14 US institutions, investigators evaluated the feasibility of testing for ten oncogenic driver mutations in patients with adenocarcinoma. Patients who were found to have mutations within nine of these oncogenes were offered targeted therapy or trial agent at their physician's discretion. Approximately onethird of the patients enrolled in the study were not tested due to insufficient tumor tissue. Of the 1017 patients tested, $72 \%$ were evaluated for all oncogenic drivers. KRAS was the most common mutation, present in $25 \%$ of tumor specimens, followed by EGFR (21\%) and ALK $(8 \%)$. Patients with an oncogenic driver who received genotype directed therapy had better survival compared with those who did not receive therapy. 
In this study, the investigators were able to successfully demonstrate the feasibility of multicenter testing of oncogenic driver mutations as well as enrollment in clinical trials for targeted agents. However, the unique characteristics of their patient sample such as a significant proportion of nonsmokers ( $40 \%)$ make it difficult to generalize their findings.

- Article written by Minal Kale \& Juan Wisnivesky
Financial \& competing interests disclosure

$J$ Wisnivesky is a member of the research board of EHE

International, has received honorarium from Merck and IMS Health, and a research grant from Aventis. The authors have no other relevant affiliations or financial involvement with any organization or entity with a financial interest in or financial conflict with the subject matter or materials discussed in the manuscript apart from those disclosed.

No writing assistance was utilized in the production of this manuscript. 